\title{
La escuela de padres y madres en 10 instituciones educativas públicas de Medellín. Una estrategia para la intervención con papás
}

Darline Arévalo Quintero

Marielly Jaramillo Arboleda

Lorena Orozco Castaño

Yudy Esnei Jiménez Muñoz

Luisa María Salazar García

María Alejandra Rendón Álvarez

Estudiantes de la Especialización en Familia de la

Universidad Pontificia Bolivariana. Cohorte XXXI 2018.

\section{Luz Natalia Ocampo Mejía}

\section{Gloria Mercedes Gómez Santa}

Aprobado:

10 de julio de 2020

Docentes curso seminario especializado: Fenómenos y contexto de la familia contemporánea 


\section{Resumen}

El ejercicio de la paternidad viene atravesando una serie de cambios que se van visibilizando en diferentes espacios de la sociedad; desde una esfera educativa, las escuelas de padres y madres han sido una herramienta para aportar en medio de dichas transformaciones que requieren orientaciones, guías, y perspectivas que contribuyan a un nuevo marco de referencia de la paternidad. El objetivo de este trabajo es identificar a través de una investigación cualitativa, referentes metodológicos que soporten los programas de escuelas de padres y madres en instituciones educativas públicas en la ciudad de Medellín, a fin de cooperar en la construcción de un modelo de intervención con papás para el sector educativo. Para esto, se analizaron 10 entrevistas a docentes de instituciones educativas públicas. Se concluye que dentro de los principales resultados se encuentra la falta de espacios de formación diferenciados para la orientación de papás, de planeación y desarrollo de las escuelas de padres y madres dentro de las instituciones, así mismo, la escasez de información bibliográfica respecto a temáticas y metodologías de las escuelas de padres y madres.

Palabras clave

Paternidad, Escuela de padres y madres, Instituciones educativas, Temáticas, Metodologías, Modelos pedagógicos.

\section{Abstract}

The paternity practice has been through a series of changes that are being visible in different spaces of the society; from an educational type of view, the School of Parents has been a tool to contribute in those transformations that require orientation, guiding, and perspectives that provide a new framework of paternity. The goal of this work is to identify through a qualitative research, methodological references that support the programs of Schools of Parents in Public Educational Institutions in the city of Medellin, to supply into the construction of a model of intervention with parents for the educational sector. To do this, there were analyzed ten interviews to teachers of Public Educational Institutions. In conclusion, among the main results we can highlight the lack of training spaces differentiated to the guidance of parents, the planning and development of the Parent School within these institutions, and also the shortage of bibliographic information on the topics and the methodology of the school of parents.

\section{Keywords}

Paternity, School of parents, Educational institutions, Topics, Methodologies, Pedagogical models. 


\section{Introducción}

El presente artículo entrega algunos resultados de la investigación Aportes interdisciplinarios para la intervención con papás en la ciudad de Medellin: Aproximaciones desde los contextos jurídico, educativo y terapéutico, adelantado por varios grupos de investigación de la Universidad Pontificia Bolivariana $(\mathrm{UPB})^{1}$. Se identifican en el texto propósitos, temáticas y metodologías que soportan los programas de Escuelas de Padres y Madres (EPM) en algunas Instituciones Educativas (IE) públicas de la ciudad de Medellín a fin de aportar a la construcción de un modelo de intervención con papás ${ }^{2}$ para el sector educativo.

Teniendo en cuenta lo anterior, se reconoce la EPM como una estrategia de tipo formativo, que posibilita la orientación y la construcción conjunta de padres respecto a los retos y necesidades que se derivan de su rol. Las EPM de acuerdo con Puente (1999) son espacios que buscan aproximar a los padres a una reflexión hacia su responsabilidad en los procesos académicos de sus hijos, y a generar acercamientos entre la IE y la familia que permitan acciones pedagógicas cercanas a las realidades de los estudiantes (pp. 1-4).

Se reconoce, históricamente, como el ejercicio de la paternidad se ha vinculado con temas económicos y laborales, donde la figura del papá ha sido relacionada con provisión y autoridad, desligando al hombre de aquellos aspectos que tienen que ver con el cuidado y la crianza de los hijos. Sin embargo, de acuerdo con el concepto de Bonino (2003), la paternidad como construcción cultural, se ve inmersa en cambios dados por los contextos sociales del país, que permiten identificar diferencias en el rol masculino, encontrándose un aumento en el tiempo y atención que los papás están dedicando a sus hijos, en actividades que tienen que ver con el acompañamiento, la formación y los procesos escolares (pp. 176-177).

1 Grupos ejecutores: Grupo de Investigación en Familia (GIF), Emoción, Cognición y Comportamiento (ECCO), Grupo de Investigaciones en Derecho, Grupo de Investigaciones en Comunicación Urbana.

2 En este artículo se utilizará la palabra papá (y su plural) para referirse al hombre en ejercicio de la paternidad y padres para incluir a papá y mamá. 
Para lograr lo planteado desde este trabajo se proponen tres categorías: paternidad, escuela de padres y madres y modelos pedagógicos, que posibiliten las bases para el análisis de la información recolectada.

\section{Paternidad}

Los diferentes cambios sociales, políticos, económicos y tecnológicos que han caracterizado el inicio del siglo XXI, han influenciado la dinámica y la estructura familiar, los roles que asume la pareja y, consecuentemente, el ejercicio de la paternidad y la maternidad.

$\mathrm{Al}$ respecto, en el contexto colombiano, es pertinente mencionar el estudio compilado por Puyana (2003), denominado Padres y madres en cinco ciudades colombianas, el cual menciona tres tendencias en la forma cómo se vive la paternidad. En este, se identifica una primera tendencia denominada tradicional, en la cual, el padre es visto como proveedor, encargado de impartir la autoridad y quien delega en la madre todas las actividades relacionadas con la crianza y el cuidado. La segunda tendencia es nombrada de transición, ya que es el momento en el que se presenta una disyuntiva entre las formas tradicionales de ser papá y las nuevas corrientes emergentes. Y, finalmente, la tendencia de ruptura, en la que se identifica una democratización de las relaciones, nuevas funciones y una crianza de los hijos que se construye por medio de arreglos parentales y no de distinciones de género.

Así mismo, se percibe en esta tendencia de ruptura unas diferencias significativas en el ejercicio de la paternidad, dándose una mayor expresión afectiva a través de besos y caricias, una impartición de la autoridad menos violenta, relaciones de género menos perjudiciales hacia la mujer y una nueva valoración de la niñez.

La tendencia actual es un papá que muestra su responsabilidad al ser más cercano, más cariñoso y presente en las necesidades de sus hijos. Los discursos revelan que hoy la paternidad va mucho más allá de la provisión económica familiar y el modelo de paternidad tradicional se presenta como reto a superar: "la paternidad legítima se vincularía con la implicación directa en las atenciones a los hijos” (Barbeta-Viñas \& Cano, 2007, p. 28). 
La perspectiva de género abona también al constante cambio que se vivencia desde la construcción social del sujeto; Kimmel (1997), citado por Menjívar (2004), considera:
A la masculinidad como un conjunto de significados siempre cambiantes, que construimos a través de nuestras relaciones con nosotros mismos, con los otros, y con nuestro mundo. Es precisamente el carácter relacional de la masculinidad lo que le brinda su carácter de género. Efectivamente, tanto la masculinidad como la feminidad son construcciones relativas; su construcción social solo tiene sentido con referencia al otro (p. 101).

Desde una postura centrada en el ser que promueve la equidad entre masculinidad y feminidad se posibilitan mayores oportunidades para la democratización de las relaciones y, en este caso, en especial, para que los papás ejerzan su rol desde sus deseos y necesidades, y no solo desde una perspectiva funcionalista; "no obstante, seguimos encontrando diferencias de género y cierta elusión en la ejecución de determinadas tareas con los hijos: aquellas que requieren mayores niveles de implicación" (Barbeta-Viñas \& Cano, 2007, p. 28).

Es un reto y, a la vez, una oportunidad, la consolidación de relaciones más horizontales entre padres e hijos, donde la base de esta misma sea el respeto, la cercanía, el apoyo económico y emocional, la vinculación en el quehacer diario de los hijos, asumiendo compromisos que demuestren el valor que implica el ejercicio de la paternidad, manteniendo su esencia y su identidad desde lo masculino, en los cuales "los padres se «maternalizan», toman a las madres y determinados elementos femeninos como referencias para su paternidad" (Barbeta-Viñas \& Cano, 2017, p. 28).

Oiberman (1998), citado por Nieri (2012), sostiene:

La paternalización es el proceso psicoafectivo por el cual un hombre realiza una serie de actividades en lo concerniente a concebir, proteger, aprovisionar y criar a cada uno de sus hijos jugando un importante y único rol en el desarrollo del mismo, distinto al de la madre (p. 1000). 
Estos acercamientos han abierto puertas para que una nueva generación de papás viva la experiencia de cargar, alimentar, acariciar, jugar y acompañar a sus hijos en otras esferas no exploradas o visibilizadas como el estar presentes en la formación y educación de sus hijos.

\section{Escuelas de padres y madres}

Para hablar sobre EPM es importante mencionar que, desde la legislación colombiana, se ha considerado la crianza de los niños, niñas y adolescentes (NNA) desde el principio de corresponsabilidad. Es así como, el Código de Infancia y Adolescencia establece que "la familia, la sociedad y el Estado son corresponsables en su atención y protección” (2006, p. 3). Lo anterior propone que la familia debe mantener un vínculo con la IE para garantizar el bienestar y protección de esta población.

Para dar soporte a tal propuesta y en aras de vincular a las familias con los procesos educativos de sus hijos, el Ministerio de Educación creó la Ley 1404 de 2010, la cual reglamenta y regula la participación de padres, madres y cuidadores en las EPM de las IE. La presente ley tiene como propósito "integrar a todos los padres y madres de familia, así como a los acudientes (...) para pensar en común, intercambiar experiencias y buscar alternativas de solución a las problemáticas que se presenten en la formación de hijos e hijas" (p. 1). Como puede observarse, el llamado que realiza la legislación colombiana es a la participación activa de los padres de familia dentro de las IE, pues la educación no es un asunto que se delegue, y debe dar respuesta a las múltiples problemáticas que se presentan en la educación y crianza de los NNA.

Es así como las EPM se convierten en espacios para que papás, mamás, cuidadores y docentes puedan compartir experiencias que aporten a la formación y cuidado de los NNA. Es oportuno nombrar algunos planteamientos de los autores Ricoy y Murias (2002), según ellos "la escuela de padres y madres es una estrategia de formación que se ofrece desde el campo de la Educación para Personas Adultas y que ubicamos dentro del campo de la Educación No Formal Socio-Participativa” (p. 171), es decir, las EPM deben tener como principio la participación activa de los asistentes. 
Finalmente, se identifica que el objetivo principal de las EPM es "incidir sobre la formación en conocimientos, valores, creencias, actitudes, habilidades, conductas del grupo, etc.” (Ricoy y Murias, 2002, p. 171). Por lo anterior, es adecuado decir que las EPM deben favorecer la formación de adultos que se encuentren en procesos de crianza.

\section{Modelos pedagógicos}

Los modelos pedagógicos apuntan a la búsqueda de entender y presentar el proceso de enseñanza aplicado en cada IE de acuerdo con los propios lineamientos institucionales, características y necesidades del contexto educativo.

Entre las definiciones que soportan el concepto de modelos pedagógicos, cabe resaltar lo dicho por Flórez (1994), citado en Galeano, Preciado, Carreño, Aguilar y Espinosa (2017), en el que sostiene:

Los modelos pedagógicos son construcciones mentales mediante las cuales se reglamenta y normativiza el proceso educativo, definiendo qué se debe enseñar, a quiénes, con qué procedimientos, a qué horas, según cuál reglamento disciplinario, a los efectos de moldear ciertas cualidades y virtudes en los estudiantes. De igual manera, plantea que estos modelos son efecto y síntoma de la concepción del mundo y de las ideologías que enmarcan la vida intelectual y la circulación de saberes filosóficos y científicos en cada sociedad históricamente determinada (párr. 3).

Desde el cuestionamiento del por qué los modelos pedagógicos pueden comprenderse como un referente para la formulación de las EPM -a partir de la concepción de hombre que se desea formar- se considera importante reconocer cómo en la época actual no todos los modelos pueden ser base o referente para planear un trabajo con la familia, esto justificado en el modelo tradicional, el cual tiene una postura estructural y limita la participación en la resolución de sus propias necesidades y demandas; dicho de otra manera, este solo tiene presente ahondar en temas de interés académico, que si 
bien busca formar jóvenes expertos, olvidan la importancia de generar pensamiento crítico y autónomo.

Por otra parte, el modelo romántico, tal como lo indica Flórez (1994), citado por Gómez y Polanía,

busca desarrollar la máxima autenticidad y libertad individual del estudiante en procura de su desarrollo natural, espontáneo y libre. Los contenidos no están elaborados previamente, sino que se desarrollan en la medida en que el alumno los solicite (2008, p. 60).

Basados en el modelo, antes descrito, se podría pensar que la cultura colombiana aún no está preparada para apoyar la formación de los padres mediante una postura abierta, por cuanto las instituciones públicas tienen como prioridad cumplir con las metas de índole normativo y, posteriormente, las universidades no están dispuestas a ajustarse al modelo de aprendizaje de los jóvenes, que vienen de establecimientos que manejan dicho modelo romántico, lo cual podría ocasionar un choque para estas generaciones.

En cuanto al modelo conductual, para Flórez (1994), citado por Gómez y Polanía (2008),

se desarrolló paralelo con la racionalización y planeación económica en la fase superior del capitalismo, bajo la búsqueda del moldeamiento meticuloso de una conducta productiva en los individuos. Su método consiste en la fijación y control de objetivos instruccionales formulados con precisión. Se trata de una transmisión parcelada de saberes técnicos mediante un adiestramiento experimental expresado como tecnología educativa. Su principal exponente es Skinner (p. 56).

De acuerdo con lo anterior, puede considerarse que las EPM, al tener mayor énfasis en la conducta y cumplimiento de la norma, se alejan de la importancia representada en el soporte emocional - motivacional que se debe impulsar en los estudiantes; por tanto, se asume el riesgo de crear barreras entre docentes, estudiantes, padres y comunidad, al no tener un equilibrio entre la norma y las relaciones dadas entre ellos. 
Por último, tanto el modelo progresista-desarrollista como el socialhumanista se consideran de valioso aporte para el acompañamiento desde una EPM, ya que promueven la participación activa en temas que impulsan el desarrollo y motivan al cambio social, útil en tiempos de indiferencia y normalización de las crisis reflejadas en los diferentes contextos que competen a la familia. Precisamente el modelo pedagógico social, según Flórez (1994), citado por Gómez y Polanía (2008),

busca el desarrollo de habilidades de pensamiento críticoreflexivo que permiten al estudiante participar activamente en procesos de transformación de la sociedad. Estimula la crítica del conocimiento, de la ciencia, sus textos y sus fuentes de manera permanente. Se fundamenta en el aprendizaje co-participativo y en la reflexión crítica de las propias creencias y juicios (p. 67).

\section{Materiales y métodos}

El presente estudio hace parte de la investigación titulada Aportes interdisciplinarios para la intervención con papás en la ciudad de Medellín: Aproximaciones desde los contextos jurídico, educativo y terapéutico. Con el fin de contribuir a uno de los objetivos de esta investigación, durante el curso Seminario especializado: Fenómenos y contexto de la familia contemporánea, de la Especialización en Familia de la UPB, se realizó un ejercicio investigativo con los siguientes objetivos: a) rastrear los propósitos de los programas de $\mathrm{EPM}$; b) enunciar las temáticas abordadas en los programas de EPM; y c) describir aspectos asociados a la metodología de los programas de EPM.

La investigación contó con una metodología cualitativa, como una forma de acercamiento a la realidad social; al respecto Quecedo y Castaño (2002) afirman que los estudios cualitativos:

intentan describir sistemáticamente las características de las variables y fenómenos (con el fin de generar y perfeccionar categorías conceptuales, descubrir y validar asociaciones entre fenómenos o comparar los constructos y postulados generados a partir de fenómenos observados en distintos contextos), así 
como el descubrimiento de relaciones causales, pero evita asumir constructos o relaciones a priori (p. 12).

Se analizaron diez entrevistas realizadas a docentes de IE del municipio de Medellín, claves para el diseño e implementación de acciones con papás, cuya muestra fue intencional más no representativa, es decir, se seleccionaron aquellos establecimientos que proporcionaran accesibilidad a la información. Para conservar la identidad personal e institucional, el grupo de investigación que rastreó la información codificó las entrevistas ${ }^{3}$. Estas fueron realizadas por estudiantes del pregrado de Trabajo Social de la UPB, inscritos al proyecto, quienes las transcribieron y cuya información fue llevada a una matriz categorial de acuerdo con los objetivos de la investigación macro.

Seguido a esto la matriz fue alimentada por estudiantes de la Especialización en Familia, para luego realizar una lectura intra e intertextual a partir de la elaboración de memos analíticos, entendiendo estos como instrumentos para la comprensión de la información obtenida (Jiménez, 2015, p. 1).

A partir de los objetivos propuestos se plantearon tres categorías conceptuales correspondientes a paternidad, escuela de padres y madres y modelos pedagógicos; también cuatro categorías de análisis: propósitos de las escuelas de padres y madres, temáticas abordadas en estos espacios, aspectos metodológicos y participación de papás en las escuelas de padres y madres, con las cuales se recogió información sobre los aspectos metodológicos de los procesos formativos y la participación de los papás en las IE.

Así, el análisis de los datos arrojó unos hallazgos y conclusiones que seguirán siendo insumos para la investigación macro y su articulación con cada una de las áreas de los ejecutores ${ }^{4}$, desde el contexto jurídico, educativo y terapéutico, con el fin de promover la participación y vinculación de los papás desde cada uno de estos escenarios en la ciudad de Medellín.

3 En adelante serán nombradas con el código respectivo de la entrevista (01 a 10) y con la sigla IP (Institución Pública).

4 Grupo de Investigación en Familia, Semillero en Familia, Ecco - Emoción, Cognición y Comportamiento, Grupo de Investigaciones en Derecho y Grupo de Investigación en Comunicación Urbana. Todos adscritos a las Universidad Pontificia Bolivariana. 


\section{Resultados y discusión}

A continuación, se describen los hallazgos encontrados, se presentan, inicialmente, los resultados referidos a los propósitos que tienen las diferentes EPM, en un segundo momento, las temáticas encontradas en el desarrollo de estos procesos de formación, en un tercer momento, las metodologías usadas para el desarrollo de estas EPM y, en un cuarto momento, la participación de los padres en dichos espacios.

\section{Propósitos de las escuelas de padres y madres}

En esta investigación, para identificar los estilos de intervención de las EPM se consideró necesario clasificar en escuelas A y escuelas B, por las diferencias marcadas entre ambas. Se identificaron como escuelas A aquellas instituciones que se caracterizan por realizar los encuentros de formación para padres por obligatorio cumplimiento y sin propósitos claros; se ubicarán como escuelas B las que se caracterizan por tener objetivos claros al momento de planear los encuentros de formación para padres, atendiendo a las necesidades del contexto.

Como se mencionó, las EPM desde el modelo de intervención A, se realizan en la IE por cumplimiento obligatorio, como afirmó 01IP "en la institución las escuelas de padres se hacen más por un cumplimiento"; adicionalmente, el entrevistado refirió que "se cumple con las escuelas de padres porque había que hacerla, pero no hay un seguimiento, para mirar cómo los padres han dado respuesta".

Con ello, se identifica que en la escuela A el sentimiento de obligación se refleja en los docentes o personas encargadas de adelantar estos procesos, sumado a la poca claridad al momento de plantear los objetivos, propósitos y metas de las EPM; según los relatos de los participantes, esto tiene el mismo efecto en los padres, es decir, asistir por cumplimiento a reuniones monótonas y catedráticas, en las cuales se limita la participación de los 
padres y cuidadores para escuchar a un "experto"5. Al respecto, una de las entrevistadas expresó que "algunos papás [padres] cuestionan y manifiestan 'hay profe, esas escuelas de padres siempre son lo mismo'” (01IP).

En este tipo de IE no hay propósitos claros al momento de planear y ejecutar los encuentros de formación para padres, teniendo como consecuencia una baja participación de las familias. Adicionalmente, los objetivos a tratar no emergen de las necesidades familiares, sino de las necesidades de la IE; y con relación a este punto uno de los entrevistados manifestó como "las escuelas de padres y madres, (...) van ligadas a un objetivo que se determinó mirando situaciones o necesidades de la comunidad educativa; otros procesos se hacen en ocasiones en el proyecto de la comunidad que uno tiene" (03IP). $\mathrm{El}$ no tener en cuenta las necesidades e intereses de las familias podría estar ligado a la baja asistencia y participación de la familia en las EPM.

En este orden de ideas, se observa cómo se empieza a difuminar el principio de corresponsabilidad que plantea la ley colombiana a través del Código de Infancia y Adolescencia, en donde al no existir sintonía entre las partes es difícil tener objetivos comunes, lo que trae consecuencias en el aprendizaje de los NNA y en la relación entre familia e IE. En este sentido Atao (2018) manifiesta:

es importante que los padres colaboren con el maestro en la educación del estudiante, puesto que el alumno pasa mitad del tiempo en la escuela y la otra mitad de tiempo en el hogar, por lo tanto, su educación debería estar guiada bajo patrones similares, no es posible que hoy en día el niño aprenda algo en el colegio y lo desaprenda en la casa, o viceversa (p. 24).

Como se puede evidenciar, es de vital importancia contar con la participación activa de los padres en las EPM, pues de la comunicación bidireccional también depende que el proceso de formación de los NNA sea más eficaz y que tanto la educación como la crianza se encuentren en consonancia la una con la otra. Como se mencionó, el panorama también trae algo de esperanza, es oportuno decir que la escuela con un modelo de intervención B se destaca por unas características diferentes.

5 Los entrevistados se refieren a los "expertos" como los profesionales encargados de realizar charlas de forma catedrática. 
Este modelo, entre sus particularidades, tiene como principio permitir la participación activa de los asistentes, centrándose en el intercambio de experiencias que faciliten a la comunidad educativa herramientas para la formación de NNA. Encontrándose el planteamiento de propósitos y objetivos claros al momento de planear y desarrollar EPM, identificando como uno de los propósitos principales "vincular al padre de familia con el estudiante (...) debe quedar claro que la educación de los niños es un deber del Estado, pero también es un derecho del padre de familia" (09IP).

Retomando los aportes de Ricoy y Murias (2002), quienes proponen que la educación para padres debe ser socio-participativa, se identifica que uno de los logros en las IE, que plantean objetivos claros, es que trabajan atendiendo las necesidades e intereses de las familias. Sobre esta situación, el entrevistado 10IP expuso: "yo creo que conocer el contexto ayuda mucho (...) Y ha sido realmente como, a través del tiempo, se ven transformaciones". Así mismo, el entrevistado reconoció que los padres deben tener mayor participación en esos espacios de formación, porque "ellos también tienen muchos que decir, ellos tienen toda una experiencia por delante". Observar las transformaciones familiares, trabajando desde las necesidades que los asistentes proponen, y permitiendo la participación activa, genera un vínculo entre padres de familia y docentes que favorece la educación y crianza de NNA.

También, es importante decir que este vínculo entre docentes y familias se ve reflejado en un mayor compromiso, como se enunció en el relato de 10IP: "yo creo que es el compromiso de cada una de nosotras (...) porque nadie nos dice que hay que querer, nadie nos dice que hay que abrazar, nadie nos dice que hay que ocuparnos (...) y tenemos que trabajar juntos”. En esta misma línea Farfán, Nieto y Pérez, afirman:

La escuela de padres y madres puede ser un espacio de reflexión y diálogo que permita estrechar la relación entre familia y escuela, y que posibilite la formación a padres y maestros para mejorar sus prácticas educativas en pro de incidir positivamente en el desarrollo de los niños (2018, p. 4).

Igualmente, es de resaltar los esfuerzos que realizan algunas IE para alcanzar los propósitos planteados, lo cual los lleva a idear diferentes estrategias para la integración de los asistentes y encontrar alternativas para el trabajo 
con familias; como dijo 10IP "acá nos sentamos a tejer y a conversar de cómo es la vida suya y usted cómo hace que los hijos suyos funcionen y los míos no, cosas tan simples como ayudar a su hijo a hacer una tarea"; otro de los entrevistados manifestó: "lo que plantea la institución es movilizar frente a algo" (01IP); además los procesos diferenciados permiten que los asistentes se empoderen más de esos espacios y optimicen los encuentros para fortalecer lazos. Es así como "hay unas profes que están con los papás [padres] y hay otras profes que están con los niños trabajando el mismo tema" (10IP). Esto nos lleva a concluir que las EPM tienen futuro siempre y cuando se ajusten a las necesidades familiares, se realicen procesos diferenciados y se contribuya a la participación activa del otro.

\section{¿Cómo determinar las temáticas en las escuelas de padres y madres?}

En primer lugar, se considera importante nombrar que, tanto en el aspecto temático como metodológico de las EPM, no se encontró información y bibliografía disponible para ser utilizados como marco referencial; por ende, el presente artículo puede servir de base para futuras investigaciones relacionadas con las EPM, y sus contenidos.

Además, las EPM de las IE incluidas en este estudio, se presume, no son rigurosas para la planeación, a lo que se refiere en la norma colombiana, debido a que los temas ejecutados carecen de consistencia de acuerdo con los objetivos contemplados en la Ley 1404 de 2010, que las regula.

Como complemento formativo que consagra la Ley General de Educación, es función de todas las Instituciones Educativas del sector público y privado, en los niveles preescolar, básica y media implementar y poner en funcionamiento el programa escuela para padres y madres, cuyo contenido debe ser instrumento que propenda por la formación en valores de los educandos y asegure una sociedad responsable dentro del contexto del Estado Social (Artículo 2, p. 1). 
No obstante, para escoger los temas se observan dos particularidades, una de ellas es que las IE se basan en las problemáticas de mayor demanda, tales como conductas suicidas, pautas de crianza, hábitos alimenticios, acompañamiento de los hijos, redes sociales, convivencia familiar, prevención de consumo de drogas, autocuidado, violencia de género, bullying, riesgos psicosociales, temas sobre proyecto de vida, proyecto artístico, manualidades, artes, entre otros. Sin embargo, algunos de estos temas no tienen continuidad y demandan poco interés en las personas que los planean y ejecutan, lo que impide el impacto necesario en el desarrollo del bienestar e integridad del grupo familiar, educativo y social. Esto figura también justificado en la respuesta emitida por uno de los docentes entrevistados "los temas o contenidos siempre se eligen de acuerdo con la dificultad que está más visible, (...) siempre lo que más afecta a la comunidad educativa es lo que se trabaja” (04IP).

Sin duda se debe reforzar la secuencia de los temas, puesto que ello además de dar formalismo y contundencia a los espacios realizados, puede generar motivación e interés en los convocados, y promover un sentido de pertinencia. El siguiente texto nos presenta una idea al respecto.

La técnica ideal es la "personalización", relacionar el tema con los intereses de los asistentes, mostrarles que les afecta e involucrarlos en la charla. Independientemente del tema y del entorno, la gente básicamente es la misma y le gusta que le hablen de los temas que le interesan (Martínez, 2001, p. 73).

Otro de los aportes sobre la importancia del tema, es el referido por Gutiérrez:

Es importante asegurarse de que el tema seleccionado corresponda a la metodología propuesta (...) Para que pueda desarrollarse es necesario que el grupo tenga "saberes" sobre el asunto en cuestión; de lo contrario, no podrían participar en la construcción (...) No habría, entonces, debate (2003, p. 245).

En consideración a la segunda particularidad, se encuentra que las IE consultadas para afianzar temas, algunas se apoyan en redes internas y otras en redes externas. En cuanto a las que se apoyan en redes internas, se tiene como referente la respuesta emitida en la entrevista de un docente, donde 
se identificó que "la escuela de padres cuenta con el apoyo de un grupo interdisciplinario conformado por psicólogo, trabajador social, fonoaudiólogo, que busca que los padres se enteren de los temas que sus hijos necesitan fortalecer" (07IP). En relación a las IE que tienen apoyos externos, uno de los entrevistados manifestó que "hay un programa con [una universidad pública], el cual se piensa como enganche y el tema es enseñarles el proceso de lectoescritura y lógico matemática a los padres analfabetas” (10IP).

$\mathrm{Al}$ respecto, igualmente, se tiene evidencia de otro docente entrevistado:

[Hay un programa de la Alcaldía] que atiende temas para la población con discapacidad o con alguna necesidad educativa en especial; igualmente, [otra universidad] les brinda soporte en cuanto a temas sobre el proceso de preparación de alimentos saludables que contribuyen a la nutrición de los estudiantes; hay [un colegio] que brinda talleres cada 15 o 20 días para los niños y, finalmente, desde Red Papaz dan formación a los padres de familia según resultados de encuestas en redes sociales y bases de datos para realizar un diagnóstico de las necesidades que se deben abarcar (09IP).

Así mismo, puede identificarse con el último docente citado, que para ejecutar las EPM, en algunos casos, los temas se eligen de manera sectorizada y focalizada; en promedio se tienen talleres específicos para primaria como son los de manejo de autoridad y cuidado del niño, hay otros para secundaria como, por ejemplo, el de riesgos psicosociales, prevención de consumo de sustancias psicoactivas y abuso sexual. En ocasiones optan por talleres generalizados y eligen temas que se dictan para ambas poblaciones (primaria y secundaria), como lo ilustra esta narrativa: "la mayoría de los temas escogidos dependen de las necesidades educativas especiales y sectorizadas por la institución" (09IP). 


\section{Aspectos metodológicos en las escuelas de padres y madres}

Cada una de las IE en lo que respecta a las EPM utilizan diferentes estrategias para la participación y motivación de los padres; ejemplo de ello son los siguientes relatos: "hay un regalo que los profesores construyen para los papás [padres] y los padres elaboran para los niños” (01IP), también enunciaron como "al finalizar un ciclo formativo se hace un evento y se les dan unos certificados" (09IP), en otra institución "realizan un canelazo donde se abre un espacio para tejer, permitiendo, a través del tejido, un momento más cálido, amable, donde se conoce al padre" (10IP), además de hacer significativas las EPM mediante asignación de nombres alusivos a fechas especiales para la familia, como son el día de la pareja y el día del abuelo.

Por esta razón, en la metodología de las EPM es necesario tener en cuenta tareas, desafíos e intereses personales en cuanto al credo, emociones y hábitos, que active la participación colectiva de los actores implicados, como lo exponen Valiño y Soto (2005):

Otro elemento que tiene mucha fuerza a la hora de decidir si una persona se adhiere a un grupo es el tema de la motivación. Para que la motivación sea más fuerte, tiene que haber la creencia entre los componentes del grupo de que su objetivo se puede lograr por medio de la acción conjunta de varias personas y no por la acción individual de una sola (p. 8).

En cuanto a los horarios de ejecución de las EPM, las instituciones concuerdan con que "se programen en horario laboral de los docentes" (01IP), y en diferentes horarios "a las seis de la mañana, a las doce del día, y a las seis de la tarde" (10IP); en particular hay una de estas instituciones que prefiere realizarlas con una periodicidad de cada dos o tres meses, junto con la entrega de notas; es importante indicar que solo una institución contempla adaptabilidad en horarios nocturnos y con ello ha obtenido cambios significativos, como lo hace evidente la entrevista 01IP, en donde se señaló como "en la noche la asistencia fue representativa y asistieron muchos papás". 
Las IE para la ejecución de las EPM implementan diversas herramientas de planeación y ejecución, como guías orientadoras donde se encuentra el objetivo, los materiales, la ambientación, publicación, socialización de las actividades y, finalmente, un espacio para observaciones. Estas guías pueden ser flexibles para cada proyecto y se diligencian iniciando el año, con metas y programas a cumplir con su respectivo responsable. En la entrevista 01IP se observó cómo se "llena un formato de asistencia y se realiza un proceso de evaluación por parte de los padres al finalizar cada sesión”. De igual modo, en otras instituciones se hacen registros, retroalimentación del tema y actas de compromiso. Seguido, para su ejecución, las IE manejan dos opciones, una de ellas prefiere hacerla por niveles de formación escolar, mientras las otras la ejecutan mediante la integración de todos los grados.

Al llegar a este punto es relevante fortalecer el aporte de los docentes entrevistados mediante la siguiente fuente:

La definición metodológica significa estructurar con un sentido estratégico toda la lógica del proceso que se quiere impulsar. Orientar y dar unidad a todos los factores que intervienen: los participantes y sus características personales y grupales, sus necesidades, sus intereses, el contexto en el que viven sus conocimientos sobre el tema, los objetivos que nos proponemos alcanzar, las etapas que hay que desarrollar para lograrlos, la secuencia temática que hay que seguir, las técnicas y procedimientos que vamos a utilizar en distintos momentos, las tareas de aplicación práctica que vamos a proponer y a evaluar (Jara, 1994, citado en Cano, 2012, p. 29).

Ahora bien, para convocar a los padres y acudientes a las EPM, las IE señalan las maneras como son citados; así lo describieron los diferentes profesores que participaron en las entrevistas: "se reparten a las personas que tienen más disponibilidad de tiempo, a las de mayor compromiso en el proceso de los estudiantes" (05IP), otro docente indicó que "se hace a aquellos estudiantes con bajo rendimiento académico y disciplinario" (06IP), también "utilizan la estrategia de voz a voz en el momento en el que los padres recogen a sus hijos" (08IP). Otras convocatorias se realizan mediante "la bitácora institucional donde se les informa las fechas de las capacitaciones, igualmente, con la entrega de notas a los padres de familia, y se les envía un 
formatico personalizado con la cita para evitar contratiempos con permisos en sus respectivos trabajos" (09IP). Del mismo modo, entre las formas más representativas de convocar a los padres y acudientes a las EPM se tiene que las instituciones hacen uso del sitio web del colegio, circulares, notas en el cuaderno de comunicaciones de los estudiantes, así como la mediación del consejo de padres, quienes intentan comunicarse con padres y acudientes. Por último, una de ellas optó por "enviar tarjetas de citación y de felicitaciones a los padres construidas por los niños" (10IP). En esta línea, Gutiérrez (2003) afirma:

Es importante que la convocatoria sea oportuna y suficiente (...) Además es primordial que la convocatoria ofrezca el programa de manera clara pero moderada. Es decir, que lo que se plantea en esta se ajuste a la realidad (...) Convocatoria por correspondencia interinstitucional, convocatoria con volante, convocatoria con plegable (pp. 250-256).

Entre las particularidades identificadas se observa que una de las IE tiene nombre asignado para la EPM, tal y como lo refirió el entrevistado 09IP: "cuenta con nombre propio Construyendo Familia". En otra de las instituciones para reforzar la asistencia y participación de los padres y acudientes se implementan estrategias de mejora, de acuerdo con lo enunciado en la entrevista 10IP: "cuando el papá [padres] no aparece, los profesores empiezan a insistir y a veces hasta los buscan donde trabajan, con el fin de investigar qué está pasando porque es un trabajo de todos”.

Cabe resaltar que dentro de las $10 \mathrm{IE}$ entrevistadas, solo tres de ellas tienden a adoptar un modelo pedagógico social - humanista, definido según McLaren (1999), citado en Gómez y Polanía:

La pedagogía social examina a las IE tanto en su medio histórico como en su medio social, por ser parte de la hechura social y política que caracteriza a la sociedad dominante. En este sentido, propende por un mayor nexo entre trabajo productivo y educación, y por el acceso a esta última de todos los individuos, sin distingo de clase social (2008, p. 66). 
Por último, los modelos pedagógicos de las siete IE restante, asumen un modelo tradicional (cuatro) y un modelo conductual (tres), prevaleciendo modelos autoritarios que dejan de lado la participación activa de los padres.

\section{Participación del papá en la EPM}

El análisis de las entrevistas permite observar la forma como los papás participan o no de las EPM, y la falta de espacios diferenciados para estos.

Se encuentra en los relatos de los docentes, que las EPM, están direccionadas para todo el grupo familiar al que pertenecen los estudiantes, sin contar con espacios de formación diferenciados para papás, pues la realidad es que las instituciones usan las EPM para tratar temas específicos que afectan a la IE directamente, dejando en un segundo plano las necesidades de las familias.

Otra de las razones que se encontraron para justificar que las IE no generan espacios de encuentro diferenciado para papás, es que los entrevistados manifiestan que la realidad de las familias ha cambiado. En los últimos años, los hogares monoparentales han aumentado, siendo la mujer la que ha quedado sola al cuidado de sus hijos (Observatorio de Políticas de las Familias, 2015, pp. 15, 26) y, por ende, son ellas las que asisten a los establecimientos educativos en calidad de acudientes. Como afirmó una de las docentes entrevistadas:

La mayoría de las familias son extensas o monoparentales, pero en su gran mayoría son las mamás las que asumen el cuidado de sus hijos, entonces nosotros no nos enfocamos en hacer distinción de género sino en hacer una atención pertinente para la formación de los estudiantes (09IP).

Según Puyana (2007) es importante comprender que uno de los cambios que ha vivido la estructura familiar durante los últimos 30 años en Colombia es el aumento de las separaciones conyugales, lo cual ha dejado muchos hogares monoparentales. Según el análisis realizado por el Observatorio de Políticas de las Familias (2015) de las encuestas de Calidad de Vida de 1993, 2003 y 2014, identifican como el hogar monoparental se constituye de manera 
creciente en una nueva forma de organización familiar, en el cual aumenta la tasa de jefatura femenina.

Es así como en las IE consultadas, se observa un elemento en común: la madre como aquella persona que asume un rol activo frente a las diferentes demandas que se hacen en los espacios escolares. Aunque son pocos los papás que asisten a las EPM, hay un grupo de ellos que valora y asume esa responsabilidad, lo cual genera resultados positivos en el desarrollo del niño. Una de las docentes afirmó:

Pues sí, para mí es súper importante que el papá está ahí. Pues su presencia, su imagen y proyección le dan al estudiante algo diferente que hace que sobresalga con el resto de los niños. Pues yo he visto papás muy presentes y se nota la diferencia entre un estudiante y otro (05IP).

Se evidencia en el análisis de las entrevistas que algunos docentes reconocen experiencias significativas de papás que asumen la paternidad, con un rol activo en los procesos educativos de sus hijos; resalta el acompañamiento y el empoderamiento. El primero se observa en el siguiente relato:

Hay uno que, por ejemplo, la esposa lo dejó con [el hijo] entonces él se encarga del niño un cien por ciento y hace lo que sea, llámese escuela de padres o reunión, él asiste y es un papá con muy buen acompañamiento (03IP).

Por otra parte, el segundo afirmó "pues nosotros aquí lo hemos visto muy distinto, es un papá empoderado, es un papá que se hace cargo, no es solo proveedor, es un papá que se ocupa de la formación, que se ocupa de la crianza" (10IP).

Podríamos identificar entonces estos casos nombrados por los docentes, donde papás asumen la paternidad desde un rol activo, que revelan la tendencia que Puyana (2003) denomina ruptura, en donde se introducen cambios con relación a las funciones paternas, además de estar direccionadas desde el rol proveedor, que se van abriendo paso a la crianza, a las relaciones afectuosas que contradicen estereotipos de autoritarismo revelados siempre 
desde el hombre (pp. 65-66). Cambios con pasos hacia adelante, que abren camino a la ruptura de lo que significó ser papá en generaciones pasadas.

Puyana (2007) expresa cómo los cambios que ha vivido el país, lo han llevado a un proceso de modernización cultural, lo cual ha implicado que dichas transformaciones incidan en la forma como los padres abordan las funciones socializadoras que la sociedad les demanda.

Quizá la ausencia de papás, en los procesos de formación que posibilitan las escuelas, está ligada a otros factores, que van más allá de la falta de interés, como es el caso de los permisos laborales y tiempos que afectan el ejercicio de su paternidad. Por ejemplo, una de las entrevistadas expresó: "entonces se les dificulta más a los padres que les den permiso en el trabajo que a las mamás, porque en muchos trabajos dicen que pida permiso la mamá, pero usted como papá debe estar aquí en el trabajo" (01IP).

\section{Conclusiones y recomendaciones}

- Se identifica que las IE que realizan las EPM por cumplimiento normativo, evidencian consigo limitaciones, tales como: poca apropiación y liderazgo de los directivos, docentes y otros profesionales a cargo de las EPM, baja asistencia y participación de los padres, que puede estar ligada a la falta de interés en las temáticas y a la percepción de que no aportan a la comprensión de problemáticas familiares.

- Se determinó como en algunas EPM las temáticas abordadas son seleccionadas de acuerdo con las problemáticas y necesidades de la IE: especialmente se tratan temas que afectan el funcionamiento y rendimiento académico de los NNA, en aras de abordar asuntos de mayor interés y que aporten a la formación de adultos. Se propone que en las EPM las temáticas surjan de las necesidades del contexto y de las familias, donde estas últimas asuman un rol más activo y vinculado.

- Conociendo las metodologías implementadas en las IE entrevistadas, se observa la necesidad de realizar cambios en la planeación de las EPM; por tanto, se sugiere que exista claridad en la planeación, identificación de propósitos y estrategias de vinculación familiar, encontrar otras maneras 
de convocar a los padres y acudientes, si se quiere lograr permanencia, lealtad, continuidad y dar significado relevante a las EPM.

- Teniendo como referente el principio de corresponsabilidad es importante que tanto las IE como el sector empresarial sean flexibles y permitan espacios de encuentro ${ }^{6}$, pues ha de reconocerse que la formación para adultos se constituye en inversión social y tiempo de calidad para formar a NNA.

- A partir de lo hallado en las entrevistas, es importante resaltar que algunas IE aportan a la diversificación del rol paterno, el cual va más allá de la provisión económica, permitiendo que el hombre se movilice y sea parte activa en el cuidado de sus hijos, evidenciándose cambios en cómo el hombre asume la crianza y tiene manifestaciones de afecto mucho más abiertas. Según los referentes teóricos, esta situación indica que algunas familias de las IE entrevistadas se están movilizando hacia las tendencias de transición y ruptura. Por lo anterior, las IE deben seguir trabajando en la apertura a la inclusión activa del papá y propiciar espacios de respeto y construcción conjunta que posibiliten un ejercicio de la paternidad desde el goce, el disfrute y la decisión genuina.

- Con el fin de contribuir a la construcción de un modelo de intervención, se observa la necesidad de realizar un giro lingüístico y utilizar un vocabulario incluyente, toda vez que a las EPM asisten diferentes cuidadores como abuelos, tíos, hermanos, primos, etc. Por consiguiente, se sugiere que las EPM pueden ser nombradas como Escuelas Familiares (EF).

- Se recomienda que docentes y demás personas que hacen parte de las EPM asuman una postura crítica que permita dar un giro significativo a estos encuentros, de manera que cobren valor y se destaquen por brindar bienestar a la comunidad educativa.

- Para realizar intervenciones familiares se recomienda que las IE utilicen modelos de corte humanista, de manera que puedan enriquecer sus particulares estilos de enseñanza y mejorar sus prácticas, con elementos de reflexión crítica sobre el hombre y la sociedad en general, y sobre su propia subjetividad en particular y así lograr un sentido propio a la hora de realizar las EPM.

- Finalmente, los apartados de Temática y Metodología en las EPM carecen de fuentes de información y bibliografía disponible para ser

6 Esto en lo referido a los horarios en que las IE programan espacios de formación para adultos y la flexibilidad que debe tener el sector empresarial para otorgar permisos a sus funcionarios. 
utilizados como marco referencial, con lo cual el presente artículo puede ser considerado como una fuente que sirva de base para continuar investigando al respecto.

\section{Referencias}

Atao, L. (2018). Los desafíos de la participación de padres de familia en las escuelas. [Tesis de grado, Universidad Inca Garcilaso de la Vega, Lima, Perú]. http://repositorio. uigv.edu.pe/bitstream/handle/20.500.11818/2647/TRAB.SUF.PROF_YERALDIN\%20 LIZET\%20ATAO\%20RAMOS.pdf? sequence $=2 \&$ isAllowed $=\mathrm{y}$

Barbeta-Viñas, M. y Cano, T. (2007). ¿Hacia un nuevo modelo de paternidad? Discursos sobre el proceso de implicación paterna en la España urbana. Revista Española de Investigaciones Sociológicas, (159), 13-30. doi: 10.5477/cis/reis.159.13

Bonino, L. (2003) Las nuevas paternidades. Cuadernos de Trabajo Social, (16), 171-182. https://dialnet.unirioja.es/servlet/articulo? codigo $=857744$

Cano, A. (2012). La metodología de taller en los procesos de educación popular. Revista Latinoamericana de Metodología de las Ciencias Sociales, 2(2), 29. http://www.memoria. fahce.unlp.edu.ar/art_revistas/pr.5653/pr.5653.pdf

Congreso de la República de Colombia. (2006, 8 de noviembre). Ley 1098. Por la cual se expide el Código de la Infancia y la Adolescencia. Diario Oficial 46.446. https://www.icbf. gov.co/cargues/avance/docs/ley_1098_2006.htm

Congreso de la República de Colombia. (2010, 27 de julio). Ley 1404. Por la cual se crea el programa escuela para padres y madres en las instituciones de educación preescolar, básica y media del país. Diario Oficial 47783. http://www.suin-juriscol.gov.co/viewDocument.asp?ruta $=$ Leyes $/ 1678836$

Farfán, R. Nieto, C. y Pérez, D. (2018). Escuela de padres para el ciclo dos de la educación básica en doce IE oficiales de las localidades de Usme y Bosa de Bogotá DC. [Tesis de maestría, Universidad de la Salle, Bogotá, Colombia] http://repository.lasalle.edu.co/ bitstream/handle/10185/24858/85161235_2018.pdf? sequence $=1$

Galeano, G.A., Preciado, M.G., Carreño, C.J., Aguilar, V.L., y Espinosa, R.O. (2017, 14 de diciembre). ¿Qué es un modelo pedagógico? Magisterio. https://www.magisterio.com.co/ articulo/que-es-un-modelo-pedagogico

Gómez, M. y Polanía, N. (2008). Estilos de enseñanza y modelos pedagógicos: Un estudio con profesores del Programa de Ingeniería Financiera de la Universidad Piloto de Colombia. [Tesis de grado, Universidad de la Salle, Bogotá, Colombia].

Gutiérrez, G. (2003). El taller reflexivo. Tercera edición. Universidad Pontificia Bolivariana. 
Flórez, R. (1994). Pedagogía del conocimiento. McGra-hill.

Jara, O. (1994). La concepción metodológica dialéctica, los métodos y las técnicas participativas en la educación popular. Centro de Estudios y Publicaciones Alforja.

Jiménez. B (2015). El memo analítico y el análisis intertextual en la investigación cualitativa. [Sin publicar]

Kimmel, M. (1997). Homofobia, temor, vergüenza y silencio en la identidad masculina. En Valdés, T. y J. Olavarría, Masculinidad: Poder y crisis (pp. 49-62). FLACSO.

McLaren, P. (1999). Pedagogía Crítica. CINDE.

Martínez, J. (2001). Aprender a comunicarse en público: Guía práctica. Paidós Ibérica.

Menjívar, M. (2004). ¿Son posibles otras masculinidades? supuestos teóricos e implicaciones políticas de las propuestas sobre masculinidad. Revista electrónica Reflexiones, 83(1), 97-106. https://revistas.ucr.ac.cr/index.php/reflexiones/article/view/11387/10742

Nieri, L.P. (2012). Sentimientos del padre actual en la etapa perinatal. Revista electrónica Iztacala, 12, 999-1022. http://www.revistas.unam.mx/index.php/repi/article/view/33642

Observatorio de Políticas de las Familias. 2015. Tipologías de Familia en Colombia: evolución 1993-2014. https://observatoriodefamilia.dnp.gov.co/Documents/Documentos\%20 de\%20trabajo/D3-tipologias-evolucion_dic3-(1).pdf

Oiberman, A. (1998). Padre-bebé: inicio de una relación. Universidad Nacional de la Plata.

Puente, De la F. (1999). Escuela de Padres: Urgencia y renovación. Padres y Maestros, (246), 1-4. http://revistas.upcomillas.es/index.php/padresymaestros/article/viewFile/2614/2313

Puyana, Y. (Comp). (2003). Padres y madres en cinco ciudades de colombianas: Cambios y permanencias. Almudena editores.

Puyana, Y. (2007). Los cambios en la paternidad y la maternidad y las formas tradicionales de castigo. En Durán, E. et al., Derechos de los niños y las niñas: debates, realidades y perspectivas (pp. 301-314). Universidad Nacional de Colombia, Facultad de Ciencias Humanas, Centro de Estudios Sociales-CES, Observatorio sobre Infancia.

Quecedo, R y Castaño, C. (2002). Introducción a la metodología de Investigación Cualitativa. Revista de psicodidáctica, (14), 5-39. https://www.redalyc.org/articulo.oa?id=17501402

Ricoy, M. C. y Murias, T. F. (2002). Estrategias de intervención para la Escuela de Padres y Madres. Educación XX1, 5(1), 171-197. http://revistas.uned.es/index.php/educacionXX1/article/view/390/340

Valiño, S y Soto, H. (2005). Manual para los grupos de Interaprendizaje - GIA. https://kupdf. net/download/gruposdeInteraprendizaje_599fa422dc0d60c75518496a_pdf 